\title{
Review
}

Journal of Innate
Immunity

\section{Innate Immune Activation in Intestinal Homeostasis}

\author{
Oliver J. Harrison Kevin J. Maloy \\ Sir William Dunn School of Pathology, University of Oxford, Oxford, UK
}

\author{
Key Words \\ Inflammatory bowel diseases - Pattern recognition \\ receptors $\cdot$ Nod-like receptor $\cdot$ Interleukin-23
}

\begin{abstract}
Loss of intestinal immune regulation leading to aberrant immune responses to the commensal microbiota are believed to precipitate the chronic inflammation observed in the gastrointestinal tract of patients with inflammatory bowel diseases (IBD), Crohn's disease and ulcerative colitis. Innate immune receptors that recognize conserved components derived from the microbiota are widely expressed by both epithelial cells and leucocytes of the gastrointestinal tract and play a key role in host protection from infectious pathogens; yet precisely how pathogenic and commensal microbes are distinguished is not understood. Furthermore, aberrant innate immune activation may also drive intestinal pathology, as patients with IBD exhibit extensive infiltration of innate immune cells to the inflamed intestine, and polymorphisms in many innate immunity genes influence susceptibility to IBD. Thus, a balanced interaction between the microbiota and innate immune activation is required to maintain a healthy mutualistic relationship between the microbiota and the host, which when disturbed can result in intestinal inflammation.

Copyright $\odot 2011$ S. Karger AG, Basel
\end{abstract}

\section{Introduction}

The lower gastrointestinal tract of mammals is inhabited by a very dense and diverse bacterial microflora that exists in a state of mutualism with the host. A constant molecular dialogue between the microbiota and the host is required for the establishment and maintenance of intestinal homeostasis. Dysregulated immune responses to the commensal microbiota and accompanying intestinal inflammation occur directly as a loss of equilibrium between finely tuned pro- and anti-inflammatory mechanisms within the gastrointestinal tract $[1,2]$. Although the intestinal mucosa is populated by a number of specialized lymphocyte populations that mediate diverse immune responses to gut pathogens and commensals, these adaptive responses are elicited following sensing of microbial or environmental factors by innate immune cells. The importance of innate immune pathways in intestinal homeostasis is underscored by genome-wide association studies that have identified polymorphisms in many innate immunity genes that influence susceptibility to inflammatory bowel disease (IBD) [3]. The innate immune compartment in the gut encompasses many innate leucocyte populations, as well as several types of intestinal epithelial cells (IEC), which act together to maintain a balanced immune response to the microbiota. Here, we review the key roles of innate immune activation in the gut, including maintenance of homeostasis during steady state, restoration of the epithelium following insult or injury, and induction of protective and pathogenic inflammatory responses.

\section{KARGER}

Fax +41613061234

E-Mail karger@karger.ch

www.karger.com
(C) 2011 S. Karger AG, Basel

Accessible online at: www.karger.com/jin
Dr. Kevin J. Maloy/Oliver J. Harrison

Sir William Dunn School of Pathology, University of Oxford

South Parks Road, Oxford OX1 3RE (UK)

Tel. +441865275589

E-Mail kevin.maloy@path.ox.ac.uk/oliver.harrison@path.ox.ac.uk 


\section{IEC Intrinsic Pattern Recognition Receptor} Activation Regulates Intestinal Homeostasis

The intestinal epithelium consists of a single layer of columnar epithelial cells that provide an effective physical barrier separating the vast bacterial load of the intestinal flora from cells of the host immune system. The continuous crypts and villi that make up the intestinal epithelium possess several physical, biochemical and immunological mechanisms ensuring intestinal homeostasis, i.e. mutualistic interactions with commensal microbes contrasted by protective immunity to invasive pathogens. Actin-rich microvillar protrusions from the apical IEC surface form a mechanical brush border, which, in combination with goblet cell-secreted mucins, comprise a sterile barrier that is impermeable to most intestinal microbes. The mucus layer of the intestine consists of an inner glycocalyx of membrane-anchored mucins, covered by an outer layer of secreted mucins, which, in addition to being a viscous barrier to microbes, forms a matrix loaded with high concentrations of IEC-derived antimicrobial peptides and secretory IgA.

IEC are permanently in contact with the intestinal lumen contents and, therefore, ideally located to undertake immunosurveillance of commensal and pathogenic populations within the intestinal microbiota. Microbe-associated molecular pattern triggering of pattern recognition receptors (PRR) classically drives a nuclear factor- $\kappa \mathrm{B}$ (NF- $\kappa \mathrm{B})$-dependent pro-inflammatory response and initiation of both innate and adaptive immune responses to the invading microbe. Triggering of PRR signalling within IEC is critical for a broad spectrum of host-protective responses to pathogenic species in the intestine, including enhanced production of cytokines, chemokines and induction of B-cell class switch recombination for the production of secretory IgA [4]. In order to discriminate between commensal and invasive pathogenic bacteria, IEC display compartmentalized expression of Toll-like receptors (TLR), as well as differential regulation of transcription networks in response to TLR ligands [4]. It is now well recognized that under steady state conditions, sensing of the microbiota and basal PRR signaling in IEC is important for intestinal homeostasis and constant renewal of the epithelial barrier. IEC intrinsic TLR triggering maintains immune pressure on the commensal flora through production of broad-spectrum anti-microbial peptides (AMP) that are able to limit both colonization and translocation of commensal and pathogenic species [4]. In addition, following infection or insult, IEC intrinsic PRR signalling promotes epithelial renewal, restitu- tion and tight junction fortification by driving expression of anti-apoptotic and proliferative genes [4]. Acute intestinal inflammation observed in the dextran sodium sulphate (DSS) colitis model arises due to a loss of epithelial integrity and disruption of barrier function between the intestinal microbiota and lamina propria immune cells. This triggers a potent inflammatory response mediated primarily by innate leucocytes, followed by restitution and repair of the epithelial barrier. Early studies using the DSS colitis model found that genetic deficiency in TLR4 or MyD88 resulted in increased IEC apoptosis and more severe intestinal pathology, demonstrating a cell autonomous role for TLR signalling in IEC proliferation and integrity. These protective functions are not only dependent on sensing of the microbiota by TLR because recent studies demonstrated that similar responses were elicited by activation of cytosolic Nod-like receptor (NLR) signalling pathways in IEC. Thus, mice deficient in NLRP3, or the associated adaptor ASC, or the inflammasome effector caspase-1, exhibited hyper-susceptibility to acute DSS colitis, with increased histopathological damage, elevated chemokine levels and leucocyte infiltration that was accompanied by increased systemic dispersion of commensal bacteria $[5,6]$. Both NLRP3 and caspase-1 deficiency resulted in significantly decreased IEC proliferation and turnover during acute colitis, and bone marrow chimera experiments demonstrated that genetic deficiency in NLRP3 in the intestinal epithelium was the major factor responsible for the exacerbated disease phenotype $[5,6]$. The protective effects of NLR signalling in IEC during DSS colitis were associated with the production of interleukin (IL)-18, a cytokine that is secreted following NLR-mediated activation of caspase-1 $[5,6]$. Consistent with these findings, a polymorphism in the human NLRP3 gene that leads to reduced NLRP3 expression was identified as a risk allele for Crohn's disease (CD) [7].

Mutations in NOD2 (CARD15), which encodes the cytosolic NLR NOD2 that detects the bacterial peptidoglycan derivative muramyl dipeptide (MDP), have long been associated with susceptibility to $\mathrm{CD}$, but the mechanisms responsible remain unclear [8]. NOD2 signalling in myeloid cells has been reported to attenuate inflammatory responses induced by TLR and may also limit IL-1 $\beta$ secretion, suggesting that constitutive detection of MDP by myeloid cells may dampen pro-inflammatory responses in the gut [8]. However, several lines of evidence suggest that intrinsic NOD2 signals also play an important role in IEC homeostasis. NOD2 has been linked to the secretion of $\alpha$-defensins by Paneth cells in the small intestine, and administration of MDP has been reported to protect 
against experimental colitis in mice [8]. Moreover, recent studies have identified a link between NOD2 and autophagy, a conserved catabolic pathway in which cytoplasmic contents are targeted for degradation in lysosomal compartments. Autophagy is upregulated in response to starvation, stress or infection and may play an important role in defence against intracellular bacteria. Polymorphisms in autophagy genes, such as IRGM and ATG16L1, have been associated with susceptibility to CD [3], and it was recently reported that activation of NOD2 could stimulate autophagy and that NOD2 directly facilitated the recruitment of ATG16L1 to sites of bacterial entry at the plasma membrane $[9,10]$. Furthermore, autophagy is closely linked to endoplasmic reticulum (ER) stress and the consequent induction of the unfolded protein response. The highly secretory nature of many IEC makes them pre-disposed to ER stress, and polymorphisms in ER stress genes are associated with IBD [11]. Taken together, these findings suggest that PRR, ER stress and autophagy circuits function in an integrated manner to regulate bacterial handling in the intestinal epithelium $[2,11]$.

NF- $\kappa \mathrm{B}$ signalling within both IEC and haematopoietic cell compartments is fundamental to the maintenance of intestinal homeostasis. Appropriate pro-inflammatory stimuli initiate signalling cascades ultimately resulting in ІкB kinase (IKK) complex-dependent activation and nuclear translocation of NF- $\mathrm{KB}$ subunit heterodimers that drive gene transcription. Selective disruption of homeostatic NF- $\mathrm{B}$ signalling within IEC, by targeted ablation of the IKK regulatory subunit NEMO $\left(\mathrm{NEMO}^{\mathrm{IEC}-\mathrm{KO}}\right)$ or of both IKK-catalytic subunits IKK $\alpha$ and IKK $\beta$, led to spontaneous IEC apoptosis and severe intestinal inflammation [12]. NEMO ${ }^{\mathrm{IEC}-\mathrm{KO}}$ mice exhibited reduced AMP secretion from IEC resulting in increased levels of commensal bacteria crossing the epithelial barrier to the lamina propria. In addition, IEC in NEMO ${ }^{\mathrm{IEC}-\mathrm{KO}}$ mice were hypersensitive to tumor necrosis factor (TNF)- $\alpha$-induced apoptosis and genetic ablation of TNFR1-ablated intestinal inflammation [12]. Similarly, mice rendered specifically deficient in RelA or IKK $\beta$ in IEC exhibited increased susceptibility to chemically induced colitis [13]. Recent evidence indicates that the NF$\kappa \mathrm{B}$ target gene A20 (TNFAIP3), which has been associated with $\mathrm{CD}$, plays a key role in preventing excessive apoptosis of IEC, as mice with selective ablation of A20 in IEC exhibited increased susceptibility to DSS colitis and to TNF- $\alpha$-triggered apoptosis [14]. Another important anti-inflammatory circuit induced by NF- $\kappa$ B signalling in IEC is the secretion of the epithelial cytokine thy- mic stromal lymphopoietin (TSLP). Microbiota sensing by IEC elicits IKK $\beta$-dependent production of TSLP that plays a key role in conditioning local dendritic cell (DC) populations within the intestine that in turn regulate the type and magnitude of effector T-cell responses in the gut [15]. The range of intestinal microbes that manipulate these pathways further illustrates the importance of NF$\kappa \mathrm{B}$ signals in regulating intestinal homeostasis. Pathogenic bacteria and viruses exhibit an expansive repertoire of microbial mechanisms aimed at immune subversion by perturbation/activation of NF- $\mathrm{KB}$ signalling. Commensal microbes may also inhibit efficient transduction of NF- $\kappa \mathrm{B}$ signals in order to temper or avoid inflammatory responses. For example, enhanced nuclear export of the active NF- $\kappa \mathrm{B}$ subunit RelA in complex with peroxisome proliferator-activated receptor- $\gamma$ drives the anti-inflammatory activity of the human anaerobic commensal Bacteroides thetaiotaomicron [16]. Taken together, these findings indicate that numerous synergistic mechanisms, both host and microbe mediated, ensure that tonic levels of intrinsic PRR signalling occur constitutively in IEC and that this maintains epithelial integrity and turnover, regulates the composition and penetrance of the microbiota, and prevents overt inflammation in response to commensals.

\section{Myeloid Cells Regulate Immune Responses in the Healthy and Inflamed Gut}

Intestinal antigen-presenting cells (APC) comprise heterogeneous cell populations that are present within the organized gut-associated lymphoid tissue, including Peyer's patches, isolated lymphoid follicles and draining mesenteric lymph nodes (MLN) and the lamina propria. Recent advances have described differences in ontogeny, phenotype and tissue location that relate to functionally distinct populations of intestinal myeloid cells, which encompass DC, monocytes and macrophages [17]. As reviewed elsewhere within this issue [18], intestinal macrophages have many adaptations to prevent overt inflammatory responses to the microbiota under homeostatic conditions; therefore, we shall mainly focus on effector functions of intestinal myeloid cells. Differential expression of fractalkine receptor CX3CR1 and integrin subunit CD103 identifies two major populations of intestinal myeloid cells that have been implicated in tolerance pathways in the intestine. $\mathrm{CD}_{11 b^{+}} \mathrm{CD}_{103^{+}}$lamina propria $\mathrm{DC}$ derive from pre-classical DC lineage, whereas $\mathrm{CD} 11 \mathrm{c}^{+}$ CX3CR $1^{+}$mononuclear phagocytes (MP) may constitute 
a more heterogenous population of DC and macrophages that stem from Ly- $6 \mathrm{C}^{+}$monocytes under homeostatic conditions [17].

Microbiota-dependent signals drive CD $11 \mathrm{c}^{+} \mathrm{CX} 3 \mathrm{CR} 1^{+}$ $\mathrm{MP}$ accumulation in the lamina propria adjacent to the IEC layer [19]. Expression of tight junction proteins by CX3CR $1^{+}$MP allows projection of transepithelial dendrites in order to sample luminal contents, and this process has been suggested to contribute to resistance to enteric pathogens, such as Salmonella typhimurium. However, $\mathrm{CD} 11 \mathrm{c}^{+} \mathrm{CX} 3 \mathrm{CR} 1^{+} \mathrm{MP}$ appear not to traffic to the draining MLN or prime naïve T cells, and the defective induction of systemic immune tolerance to dietary antigen (oral tolerance) observed in CX3CR1-deficient mice instead supports a role for $\mathrm{CX} 3 \mathrm{CR} 1^{+} \mathrm{MP}$ in enhancing tolerogenic immune responses in the gut [20]. Impaired induction of oral tolerance was associated with a failure to expand lamina propria FoxP3 ${ }^{+} \mathrm{T}_{\text {reg }}$ cells and with reduced production of the anti-inflammatory cytokine IL10 by intestinal myeloid cells [20]. An important role for myeloid cell-derived IL-10 in the regulation of intestinal immune responses was further supported by an independent report that an innate source of IL-10 was required for maintenance of FoxP3 expression and control of experimental colitis by FoxP3 ${ }^{+} \mathrm{T}_{\text {reg }}$ cells [21]. Thus, although FoxP3 ${ }^{+} \mathrm{T}_{\text {reg }}$ cells could suppress colitis induced by adoptive transfer of naïve $\mathrm{CD} 4^{+} \mathrm{T}$ cells into $\mathrm{Rag}^{-/-}$recipients, they were unable to prevent colitis induction in $\mathrm{Rag}^{-/-}$IL-10 ${ }^{-/-}$recipients [21]. Innate IL-10 secretion by $\mathrm{CD} 1 \mathrm{~b}^{+} \mathrm{CD} 11 \mathrm{c}^{+} \mathrm{F} 4 / 80^{+}$myeloid cells was necessary to maintain FoxP3 expression and suppressive functions of $\mathrm{T}_{\text {reg }}$ cells in the inflamed intestine [21].

In contrast to the sub-epithelial localization of $\mathrm{CX} 3 \mathrm{CR} 1^{+} \mathrm{MP}$, the anatomical location of $\mathrm{CD} 11 \mathrm{~b}^{+} \mathrm{CD} 103^{+}$ $\mathrm{DC}$ is more varied, with populations dispersed both throughout the gut-associated lymphoid tissue and lamina propria [20]. Small intestinal lamia propria $\mathrm{CD}_{103^{+}}$ DC perform typical DC sentinel functions; they can sense and take up a wide variety of foreign bacteria and innocuous antigens and migrate to the draining MLN where they initiate adaptive immune responses with an intestinal tropism, such as imprinting of the gut-homing receptors CCR 9 and $\alpha_{4} \beta_{7}$ on activated T cells and induction of intestinal B-cell class switch to IgA production [17, $20,22]$. These activities are dependent on retinoic acid (RA), a dietary metabolite derived from vitamin A, and $\mathrm{CD} 13^{+}$lamina propria DC express aldh1a1 and aldh1a2 genes that encode for retinal metabolizing enzymes required for RA synthesis [22]. CD103 ${ }^{+} \mathrm{DC}$ play an important role in inducing tolerance to intestinal antigens un- der steady state conditions, as they migrate to the MLN and promote the generation of FoxP3 ${ }^{+}$inducible $\mathrm{T}_{\text {reg }}$ cells through a transforming growth factor (TGF)- $\beta$ and RAdependent mechanism [22]. Interestingly, recent evidence indicates that distinct myeloid cell compartments may co-operate to maintain intestinal tolerance as robust oral tolerance was associated with further expansion of gut-homing FoxP3 ${ }^{+} \mathrm{T}_{\text {reg }}$ cells in the intestinal lamina propria through interactions with CX3CR1 $1^{+} \mathrm{MP}$ [23]. Taken together, these findings highlight that a number of heterogeneous subsets of myeloid cells can participate in the maintenance of immune tolerance in the gut (fig. 1a).

Myeloid cells, granulocytes and innate lymphoid cells (ILC) all accumulate within the intestine during chronic intestinal inflammation and contribute to the excessive production of pro-inflammatory mediators that drive intestinal pathology $[1,2]$. In contrast to the protective effects of 'tonic' PRR signals in IEC described above, several studies have reported that persistent PRR signals in leucocyte populations are crucial for the induction and perpetuation of chronic intestinal inflammation. For example, the microbiota-dependent spontaneous colitis that develops in IL-10 $0^{-/-}$mice was completely absent in IL-10 ${ }^{-/-}$MyD88 $8^{-/-}$mice, and genetic ablation of MyD88 also rescued the spontaneous colitis phenotype of mice harbouring an IEC-specific deletion of the IKK-regulatory subunit NEMO [12], suggesting that functional TLR signals were required for intestinal inflammation. In addition, the innate immune-meditated typhlocolitis that develops following infection of $\mathrm{Rag}^{-/-}$mice with the Gram-negative intestinal bacterium Helicobacter hepati-

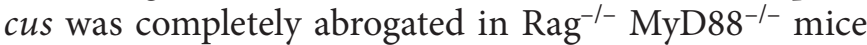
[24]. Furthermore, bone marrow chimeric $\mathrm{Rag}^{-/-}$mice in which MyD88 signalling was selectively absent in haematopoietic cells were also completely resistant to $H$. hepaticus-induced intestinal pathology, indicating a specific requirement for this signalling pathway in leucocytes [24]. Similarly, selective IKK $\beta$ deficiency in myeloid cells $\left(\right.$ LysM $^{\text {Cre }} \times \mathrm{IKK}^{\text {flox/flox }}$ ) ameliorated intestinal inflammation in the $\mathrm{IL}-10^{-/-}$model of spontaneous colitis, whereas IEC-specific deletion of IKK $\beta$ (Villin ${ }^{\mathrm{Cre}} \times$ IKK $\beta^{\text {flox/flox }}$ did not ameliorate the disease in this setting [13]. In other T-cell colitis models, it was shown that Tcell intrinsic MyD88 signals were not essential for the induction of intestinal pathology [24]; however, MyD88 signals in DC were required for the induction of pathogenic T-cell responses in the gut [25]. Together, these studies strongly support a crucial role for MyD88 signalling in myeloid cells in driving innate and adaptive inflammatory responses in the gut. During ongoing inflammation, 
Fig. 1. Innate immune circuits control intestinal homeostasis. a During steady state conditions, a number of specialized myeloid cell populations act in concert with IEC and dietary metabolites such as RA to promote tolerance to luminal antigens. b In IBD, there is a dramatic alteration in the composition and functions of myeloid cells in the intestinal lamina propria. They produce elevated levels of inflammatory cytokines that drive the activation of additional populations of innate and adaptive leucocytes, resulting in chronic inflammatory pathology. TSLP = Thymic stromal lymphopoietin; sIgA = secretory IgA.

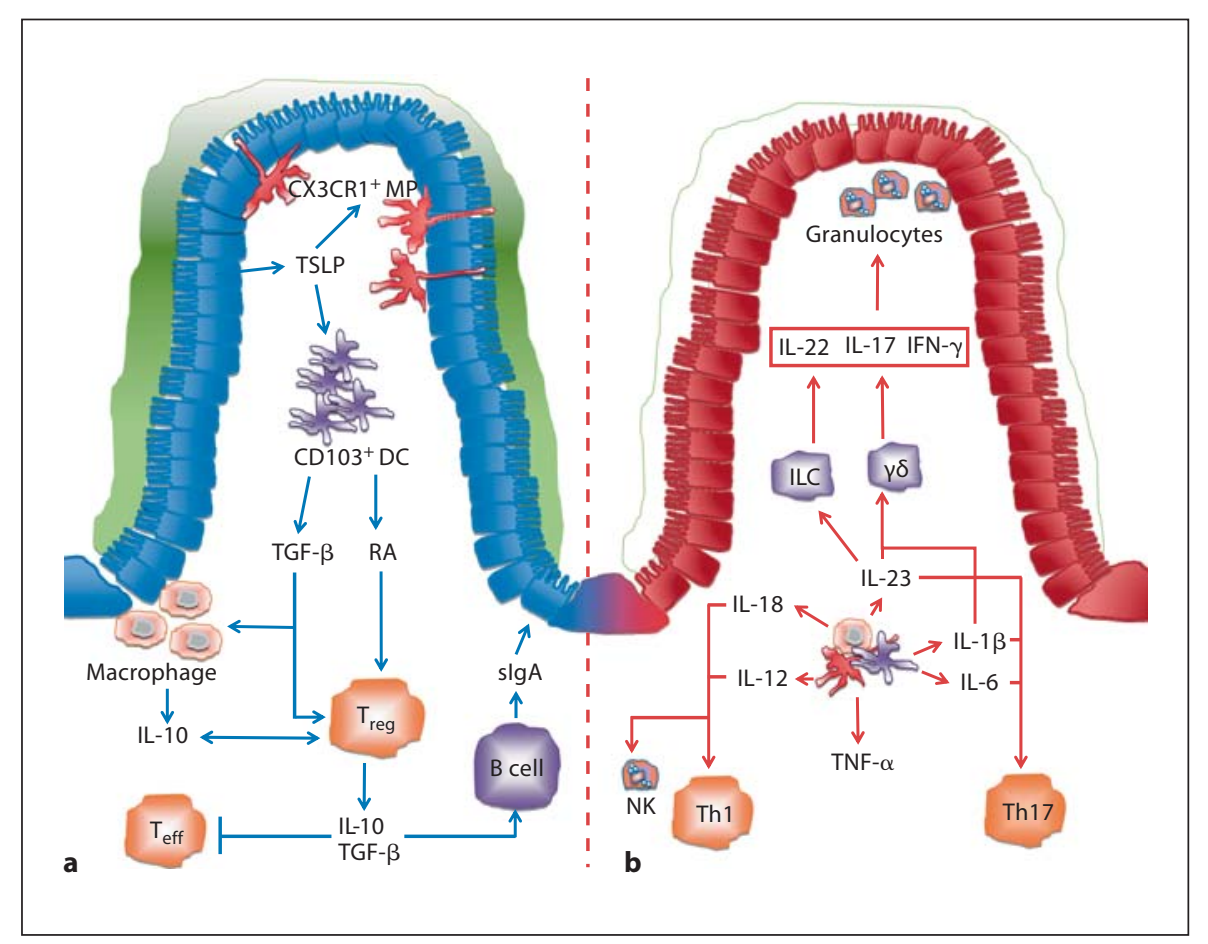

populations of intestinal myeloid APC develop pro-inflammatory characteristics that exacerbate immune-mediated pathology. Intestinal inflammation abrogates the tolerogenic activities of $\mathrm{CD} 103^{+}$MLN DC, with decreased expression of aldh1a2 and tgf $\beta 2$ correlating with an inability to promote FoxP3 ${ }^{+} \mathrm{T}_{\text {reg }}$ responses [26]. Instead, under inflammatory conditions, $\mathrm{CD}_{103^{+}} \mathrm{DC}$ produce significantly more IL- 6 than their steady state counterparts and promote the emergence of Th1 cells [26]. Similarly, flagellin-mediated activation of $\mathrm{CD} 103^{+}$lamina propria DC through TLR5 promotes the generation of Th17 cells [27]. Furthermore, a CD70 ${ }^{\mathrm{Hi}} \mathrm{CX} 3 \mathrm{CR} 1^{+}$subset of intestinal APC can detect microbiota-derived ATP through purinergic $\mathrm{P}_{2} \mathrm{X}$ and $\mathrm{P}_{2} \mathrm{Y}$ receptors, resulting in the up-regulation of molecules associated with Th17 lineage commitment, including IL-6, IL-23 and the TGF- $\beta$ activating integrin $\alpha_{V} \beta_{8}$ [28]. The same study reported that administration of an ATP analogue led to an increase in frequency of colonic Th17 cells in germ-free animals and also exacerbated T-cell transfer colitis, suggesting a role for intestinal myeloid cells in driving colonic Th17 generation under inflammatory conditions [28]. Mouse models of colitis suggest that the altered phenotypic and functional properties of myeloid cells in the inflamed intestine may partly reflect the accumulation of newly recruited cells, particularly monocyte-derived MP that respond to TLR stimulation by the elaboration of pro-inflammatory cytokines [17]. These observations are consistent with findings that intestinal tissues from CD patients exhibit infiltration by a population of $\mathrm{CD} 14^{+}$inflammatory monocytes that produce elevated levels of IL23, TNF- $\alpha$ and IL-6 [29]. A better understanding of how inflammatory myeloid cells are recruited and activated in the intestine may facilitate new therapeutic approaches in IBD.

\section{Innate Immune Cytokine Networks Drive Intestinal Pathology}

A large body of clinical and experimental evidence implicates innate leucocytes, especially myeloid cells, as critical sources of a range of inflammatory cytokines in the inflamed intestine [1]. These may exacerbate pathological responses in several ways: through effects on tissue stromal cells and IEC, through stimulation of inflammatory responses from other innate leucocytes, or through the induction of pathogenic effector T-cell responses (fig. 1b).

Blockade or neutralization of these factors constitutes an important new therapeutic approach, as exemplified by the successful application of anti-TNF- $\alpha$ antibodies in 
IBD patients [1]. Immunohistochemical studies identified lamina propria macrophages as a key source of TNF$\alpha$ in the inflamed gut, although other cells including Paneth cells and Th1 cells may also produce TNF- $\alpha$ [1]. TNF- $\alpha$ signalling can modulate a broad range of cellular responses in both haematopoietic cells and parenchymal cells, including cellular activation and proliferation, as well as potent effects on IEC integrity and apoptosis. Genetic ablation or antibody-mediated blockade of TNF- $\alpha$ has been shown to reduce or ameliorate intestinal pathology in a wide range of mouse models of IBD, indicating that it is a central factor in intestinal inflammation [1]. Treatment with anti-TNF- $\alpha$ can restore intestinal barrier function in IBD patients and this has been ascribed to reduced apoptosis of IEC together with induction of apoptosis of lamina propria mononuclear cells expressing membrane-bound TNF- $\alpha$ [1].

IL-6 is another classical pro-inflammatory cytokine that is elevated in the inflamed intestinal mucosa of IBD patients and is predominantly produced by innate leucocytes in the lamina propria [30]. IL-6 can signal both in cis-, through membrane-bound IL-6R, and in trans-, through soluble IL-6R (sIL-6R)/IL-6 complex binding to membrane-associated gp130. Thus, as gp130 is widely expressed, IL-6 potentially affects a broad range of cell types, which may have complex effects on intestinal homeostasis. For example, IEC express the IL-6R, and binding of IL- 6 may trigger cytoprotective NF- $\kappa \mathrm{B}$ activation in IEC. By contrast, increased levels of IL- 6 in the inflamed mucosa may exacerbate inflammation in several ways, such as induction of additional inflammatory mediators from macrophages and by promoting the differentiation and survival of pathogenic Th1 and Th17 cells $[1,30]$. Consistent with a dominant pathogenic role of IL-6 in IBD, beneficial effects were reported in a pilot trial of an anti-IL-6R antibody in patients with active CD [1].

Recent findings linking genetic mutations associated with secretion of the cytokines IL-1 $\beta$ and IL-18 with IBD susceptibility have rekindled interest in their roles in intestinal homeostasis. Upon activation by infection or stress, several cytosolic NLR are able to form multi-molecular platforms, termed 'inflammasomes', that drive auto-catalytic activation of caspase-1, a cysteine protease required for processing of pro-IL-1 $\beta$ and pro-IL-18 to their secreted active forms. Polymorphisms in NLRP3, IL-18 and IL-18RAP have all been associated with susceptibility to IBD [1], and both IL-1 $\beta$ and IL-18 expression are increased in inflamed IBD tissues [31,32]. Interestingly, myeloid cells lacking the IBD risk-associated autophagy gene Atg16l1 produced increased amounts of IL-
$1 \beta$ and IL-18, suggesting that a functional autophagy pathway may limit inflammasome activation. Levels of IL- $1 \beta$ are elevated in models of intestinal inflammation and blockade of IL-1 $\beta$ signalling, using anti-IL-1 $\beta$ or, by administration of the natural antagonist IL-1ra, can ameliorate the disease $[1,31]$.

IL- $1 \beta$ is secreted primarily from monocytic cells in the lamina propria and promotes activation of myeloid cells as well as neutrophilia and neutrophil migration into inflamed tissues [31]. IL-1 $\beta$ can also enhance pathogenic adaptive responses, particularly Th17 cell responses [33]. IL-18 levels are increased in several models of colitis and antibody-mediated neutralization of IL-18, or administration of the natural antagonist IL-18BP can reduce intestinal pathology [32]. In the chronically inflamed intestine of IBD patients, IL-18 is predominately associated with mononuclear leucocytes in the lamina propria, where it may contribute to disease progression through promotion of Th1 responses [32, 33]. However, the role of IL-18 in intestinal homeostasis is complex because, as noted earlier, NLR activation has been associated with protective responses in IEC. Indeed, production of IL-18 within the healthy gastrointestinal tract appears restricted to the intestinal epithelium [32], and administration of exogenous IL-18 protected NLRP3-deficient mice from exacerbated pathology during DSS colitis, suggesting an IEC-intrinsic role for IL-18 in intestinal epithelial homeostasis $[5,6]$. Similarly, genetic deficiency in either IL18 or IL-18R renders mice more susceptible to DSS colitis [32]. Thus, depending on the cellular location and temporal characteristics of activation, inflammasome-dependent cytokine responses may have beneficial or harmful effects on intestinal homeostasis [32]. Following epithelial damage, IL-18 signalling in the intestinal epithelium plays a key role in maintaining epithelial integrity, by promoting restitution and repair pathways in IEC. Conversely, chronic excessive production of IL-1 $\beta$ and IL-18 by lamina propria mononuclear cells may contribute to the deleterious inflammatory responses observed in IBD patients. Additional studies on inflammasome activation in the gut may offer further insights into IBD pathophysiology.

\section{IL-23 Drives Conserved Cytokine Circuits in Intestinal Inflammation}

Genome-wide association studies associating polymorphisms in the IL-23R and IL-12p40 with susceptibility to $\mathrm{CD}$, together with experimental studies showing 
that removal or blockade of IL-23 could ameliorate chronic colitis, have highlighted IL-23 as a central regulator of intestinal homeostasis and inflammation $[1,2]$. Production of IL-23 in the intestine is induced by PRR stimulation of myeloid cells, and DC appear to be a key source of IL-23 both during steady state conditions and also in the inflamed intestine [34]. In addition, a population of CD14 ${ }^{+}$ intestinal macrophages from the gut of $\mathrm{CD}$ patients have been reported to secrete large amounts of IL-23 [29]. IL-23 can drive intestinal pathology through excessive activation of innate and adaptive inflammatory responses $[1,2]$. IL-23 drives T-cell-dependent intestinal inflammation through direct signalling into $\mathrm{CD} 4^{+} \mathrm{T}$ cells to drive the proliferation and accumulation of effector Th1 and Th17 cells in the gut, as well as by promoting the emergence of a population of IL-17A $\mathrm{A}^{+}$interferon (IFN) $-\gamma^{+} \mathrm{CD} 4^{+} \mathrm{T}$ cells and restraining the differentiation of Foxp $3^{+} \mathrm{T}_{\text {reg }}$ cells [34]. A population of $\mathrm{CD} 161^{+} \mathrm{CD} 4^{+} \mathrm{T}$ cells able to respond directly to IL-23 stimulation with production of both IL$17 \mathrm{~A}$ and IFN- $\gamma$ has also been identified in the lamina propria of patients with IBD [35]. In addition to $\mathrm{CD} 4^{+} \mathrm{T}$ cells, several populations of innate leucocytes are able to produce IL-17A, IL-22 and IFN- $\gamma$ in response to IL-23. For example, $\gamma \delta$ T cells are enriched among the intestinal epithelial lymphocytes and can respond to IL-23 and IL-1 $\beta$ by secretion of IL-22 and IL-17A [36]. However, although $\gamma \delta \mathrm{T}$ cells have been shown to drive intestinal inflammation in some experimental models [37], their contribution to IBD remains unclear. Recent studies from several groups have identified multiple populations of novel ILC that are present in the gut and have been implicated in intestinal homeostasis, inflammation and host protection [38]. The ontogeny of different ILC populations is discussed elsewhere in this issue [39], but many appear to derive from a common ROR $\gamma \mathrm{t}^{+}$precursor that is dependent upon both cytokine and commensal bacteria-derived signals for further lineage commitment [38]. Using a mouse IBD model in which infection of $R a g 2^{-/-}$mice with $H$. hepaticus drives an IL-23-dependent innate immune-mediated typhlocolitis, we identified a novel population of $\mathrm{CD}^{-}{ }^{-} \mathrm{ROR} \gamma \mathrm{t}^{+} \mathrm{Thyl}^{\mathrm{Hi}} \mathrm{Sca}-1^{+}$ILC that accumulated in the inflamed intestine and secreted IL-17A, IL-22 and IFN- $\gamma$ in response to IL-23 [40]. Furthermore, neutralization of either IL-17A or IFN- $\gamma$ significantly attenuated colitis, and depletion of ILC using anti-Thyl completely abrogated intestinal inflammation [40].

Although IL-17A is greatly increased in the intestinal mucosa of IBD patients, experimental models have generated conflicting evidence over whether it primarily acts as a pro- or anti-inflammatory factor in the gut $[1,2]$. For example, IL-17A appears to have a protective role in acute DSS colitis whereas it appears to have a pro-inflammatory role in trinitrobenzene sulfonic acid colitis $[1,2]$. Again, these discrepancies may reflect differences in the location and temporal characteristics of IL-17A secretion, as well as in differential activities of IL-17A depending on the presence or absence of other cytokines in the local microenvironment. The pro-inflammatory effects of IL-17A have been related to the induction of cytokines and chemokines from tissue cells, such as epithelial and endothelial cells, which in turn promotes the recruitment and activation of neutrophils [2]. By contrast with the ambiguous activities of IL-17A, the pro-inflammatory effects of IFN- $\gamma$ in the intestine have been firmly established by the disease-attenuating effects of removal or blockade of IFN- $\gamma$ in experimental models of chronic colitis [1]. In the inflamed intestine, IFN- $\gamma$-secreting cells are far more prominent than those producing IL-17A, and IFN- $\gamma$ may exert pro-inflammatory effects by directly influencing IEC turnover and epithelial integrity and by activating macrophages in the lamina propria $[1,2]$. Nevertheless, the disappointing efficacy of IFN- $\gamma$ blockade in human IBD suggests that there is a complex interplay between 'Th1' and 'Th17' cytokine responses in the gut and that therapies targeting both axes may be required to effectively combat chronic intestinal pathology.

Finally, IL-23 is also a potent driver of IL-22, an IL-10 family member that acts to enhance innate immune defences in mucosal tissues [41]. Restricted to expression in the non-haematopoietic system, the IL-22R is highly enriched on mucosal epithelial cells, especially those of the skin, lung and gut [41]. In IEC, IL-22 signalling contributes to intestinal homeostasis by activation of STAT3, which enhances epithelial healing and regeneration, restores goblet cells and mucus production and stimulates the production of AMP [41]. Consistent with these observations, IL-22 administration attenuated disease severity in the DSS and T-cell receptor- $\alpha^{-/}$colitis models [41]. Furthermore, an IL-23-dependent innate source of IL-22 was shown to be critical for early immunity to the attaching/effacing mouse intestinal bacterial pathogen Citrobacter rodentium, and recent data suggest that this protective IL-22 is derived from ROR $\gamma \mathrm{t}^{+} \mathrm{Thy}^{+}$ILC [42]. Conversely, clinical evidence suggests that IL-22 may be playing a pathogenic role in IBD, as patients with active CD have elevated levels of IL-22 detectable in the serum, and levels of IL-22 correlate with disease severity and IL$23 \mathrm{R}$ genotype [2]. However, whether elevated IL-22 is driving disease progression or reflects a failed attempt at promoting resolution remains unclear. 
Fig. 2. Balanced innate immune activation maintains intestinal homeostasis. In the healthy intestine, constitutive tonic activation of innate immune pathways maintains epithelial barrier integrity and the production of innate immune factors in the epithelium. Deficiencies in innate pathways may predispose to infection or impair barrier function leading to increased bacterial translocation that elicits inflammatory responses. Conversely, chronic hyper-activation of innate immune pathways in lamina propria leucocytes plays a key role in sustaining pathological inflammatory responses in the gut. sIgA = Secretory IgA; MAMP = microbeassociated molecular pattern.

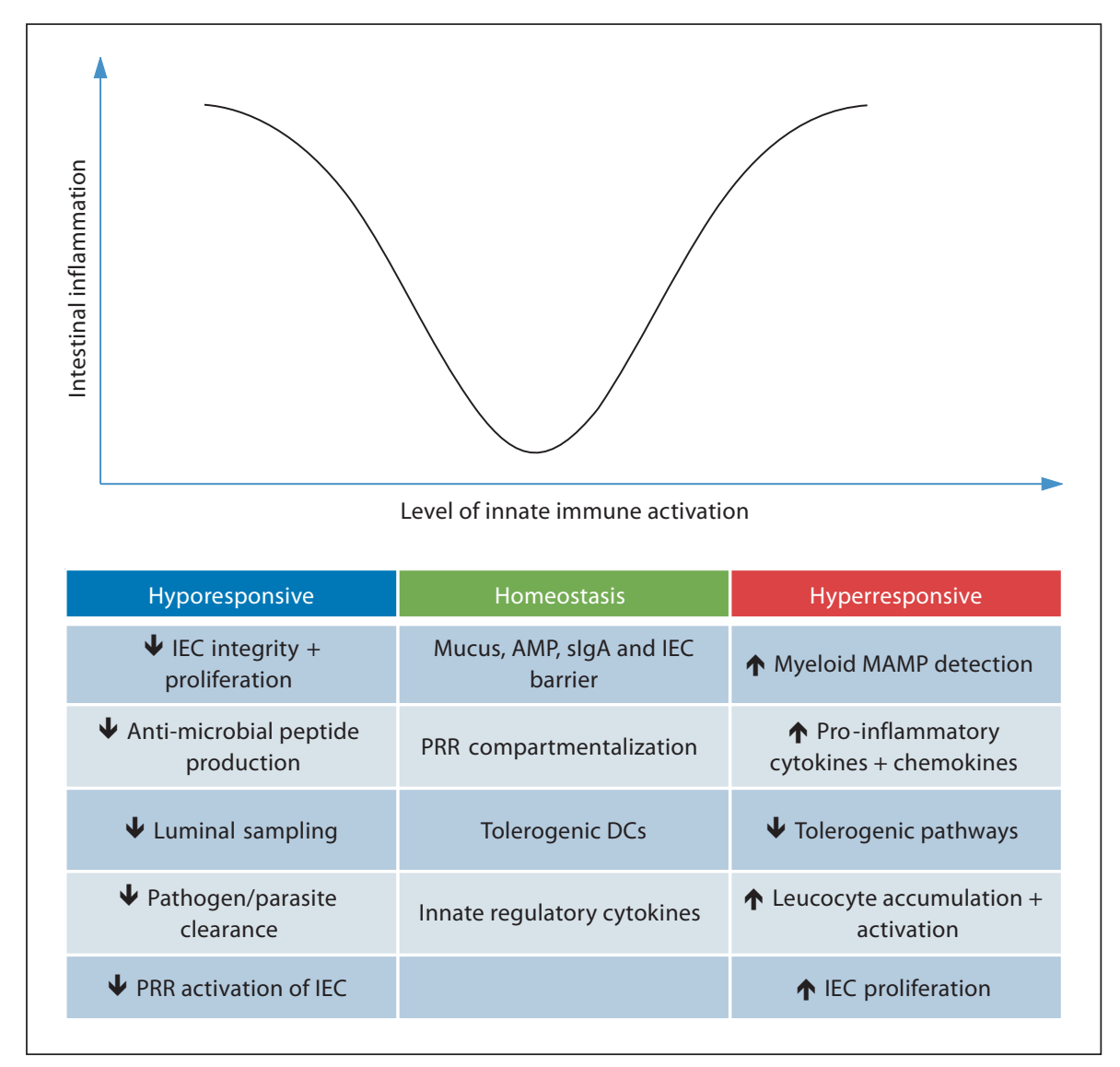

Thus, IL-23 can modulate the activation of a variety of innate and adaptive immune cells in the gut, which are mirrored by the diverse range of cytokine responses that are induced by IL-23. Under steady state conditions, these responses may play a critical role in the maintenance of epithelial barrier integrity and innate immune defence. However, sustained activation of IL-23-driven effector pathways may underlie the chronic immune pathology found in IBD (fig. 1b).

\section{Conclusions}

The data summarized in this review highlight that innate immune activation is a fundamental process that impacts on intestinal homeostasis on several levels (fig. 2). It occurs not only in haematopoietic cells, but also in parenchymal cells such as IEC. Constitutive tonic sensing of microbial factors by PRR in IEC and in innate leucocytes stimulates tissue-protective innate defences that inhibit colonization and invasion by pathogens. In the steady state, myeloid cells control circuits that prevent harmful immune responses towards that intestinal microbiota. However, damage, stress or breach of the epithelium activates a plethora of innate immune mechanisms and downstream inflammatory responses, and chronic aberrant activation of these innate immune pathways may play a key role in the characteristic pathology found in IBD patients. The activities of individual factors produced by innate immune cells in the gut, such as cytokines, may be largely determined by their location, kinetics and the presence of additional factors that can modulate their activity. Therefore, selective modulation of innate immune activation and of downstream mediators presents an ongoing challenge to effectively target deleterious inflammatory responses in IBD whilst sparing host-protective immunity in the intestinal tissues.

\section{Acknowledgement}

O.J.H. and K.J.M. are supported by funding from the Wellcome Trust. 


\section{References}

$>1$ Kaser A, Zeissig S, Blumberg RS: Inflammatory bowel disease. Annu Rev Immunol 2010;28:573-621.

$\checkmark 2$ Maloy KJ, Powrie F: Intestinal homeostasis and its breakdown in inflammatory bowel disease. Nature 2011;474:298-306.

$>3$ Franke A, et al: Genome-wide meta-analysis increases to 71 , the number of confirmed Crohn's disease susceptibility loci. Nat Genet 2010;42:1118-1125.

$\checkmark 4$ Cario E: Toll-like receptors in inflammatory bowel diseases: a decade later. Inflamm Bowel Dis 2010;16:1583-1597.

$\checkmark 5$ Dupaul-Chicoine J, et al: Control of intestinal homeostasis, colitis, and colitis-associated colorectal cancer by the inflammatory caspases. Immunity 2010;32:367-378.

6 Zaki MH, et al: The NLRP3 inflammasome protects against loss of epithelial integrity and mortality during experimental colitis. Immunity 2010;32:379-391.

$>7$ Villani AC, et al: Common variants in the NLRP3 region contribute to Crohn's disease susceptibility. Nat Genet 2009;41:71-76.

$>8$ Saleh M, Trinchieri G: Innate immune mechanisms of colitis and colitis-associated colorectal cancer. Nat Rev Immunol 2011;11: 9-20.

$>9$ Cooney R, et al: NOD2 stimulation induces autophagy in dendritic cells influencing bacterial handling and antigen presentation. Nat Med 2010;16:90-97.

$\checkmark 10$ Travassos LH, et al: Nod1 and Nod2 direct autophagy by recruiting ATG16L1 to the plasma membrane at the site of bacterial entry. Nat Immunol 2010;11:55-62.

11 Fritz T, et al: Crohn's disease: NOD2, autophagy and ER stress converge. Gut 2011, E-pub ahead of print

12 Nenci A, et al: Epithelial NEMO links innate immunity to chronic intestinal inflammation. Nature 2007;446:557-561.

13 Eckmann L, et al: Opposing functions of IKKbeta during acute and chronic intestinal inflammation. Proc Natl Acad Sci USA 2008; 105:15058-15063.

14 Vereecke L, et al: Enterocyte-specific A20 deficiency sensitizes to tumor necrosis factor-induced toxicity and experimental colitis. J Exp Med 2010;207:1513-1523.
15 Zaph C, et al: Epithelial-cell-intrinsic IKKbeta expression regulates intestinal immune homeostasis. Nature 2007;446:552-556.

16 Rahman MM, McFadden G: Modulation of NF-kappaB signalling by microbial pathogens. Nat Rev Microbiol 2011;9:291-306.

17 Varol C, Zigmond E, Jung S: Securing the immune tightrope: mononuclear phagocytes in the intestinal lamina propria. Nat Rev Immunol 2010;10:415-426.

18 Mowat AM, Bain CC: Mucosal macrophages in intestinal homeostasis and inflammation. J Innate Immun 2011;3:550564.

19 Niess JH, Adler G: Enteric flora expands gut lamina propria CX3CR1+ dendritic cells supporting inflammatory immune responses under normal and inflammatory conditions. J Immunol 2010;184:2026-2037.

20 Schulz O, et al: Intestinal CD103+, but not CX3CR1+, antigen sampling cells migrate in lymph and serve classical dendritic cell functions. J Exp Med 2009;206:3101-3114.

21 Murai M, et al: Interleukin 10 acts on regulatory $\mathrm{T}$ cells to maintain expression of the transcription factor Foxp3 and suppressive function in mice with colitis. Nat Immunol 2009; 10:1178-1184.

22 Coombes JL, Powrie F: Dendritic cells in intestinal immune regulation. Nat Rev Immunol 2008;8:435-446.

23 Hadis $U$, et al: Intestinal tolerance requires gut homing and expansion of FoxP3+ regulatory $\mathrm{T}$ cells in the lamina propria. Immunity 2011;34:237-246.

24 Asquith MJ, et al: Pathogenic and protective roles of MyD88 in leukocytes and epithelial cells in mouse models of inflammatory bowel disease. Gastroenterology 2010;139:519529.e2.

25 Feng T, et al: Microbiota innate stimulation is a prerequisite for $\mathrm{T}$ cell spontaneous proliferation and induction of experimental colitis. J Exp Med 2010;207:1321-1332.

26 Laffont S, Siddiqui KR, Powrie F: Intestinal inflammation abrogates the tolerogenic properties of MLN CD103+ dendritic cells. Eur J Immunol 2010;40:1877-1883.

27 Uematsu S, et al: Regulation of humoral and cellular gut immunity by lamina propria dendritic cells expressing Toll-like receptor 5. Nat Immunol 2008;9:769-776.
28 Atarashi K, et al: ATP drives lamina propria $\mathrm{T}(\mathrm{H}) 17$ cell differentiation. Nature 2008;455: 808-812.

29 Kamada N, et al: Unique CD14 intestinal macrophages contribute to the pathogenesis of Crohn disease via IL-23/IFN-gamma axis. J Clin Invest 2008;118:2269-2280.

30 Hruz P, Dann SM, Eckmann L: STAT3 and its activators in intestinal defense and mucosal homeostasis. Curr Opin Gastroenterol 2010;26:109-115.

31 Dinarello CA: Immunological and inflammatory functions of the interleukin-1 family. Annu Rev Immunol 2009;27:519-550.

32 Siegmund B: Interleukin-18 in intestinal inflammation: friend and foe? Immunity 2010 ; 32:300-302.

33 Zhu J, Yamane H, Paul WE: Differentiation of effector CD4 T cell populations $\left({ }^{*}\right)$. Annu Rev Immunol 2010;28:445-489.

34 Ahern PP, et al: Interleukin-23 drives intestinal inflammation through direct activity on T cells. Immunity 2010;33:279-288.

35 Cosmi L, et al: Human interleukin 17-producing cells originate from a CD161+CD4+ T cell precursor. J Exp Med 2008;205:19031916.

36 Sutton CE, et al: Interleukin-1 and IL-23 induce innate IL-17 production from gammadelta T cells, amplifying Th17 responses and autoimmunity. Immunity 2009;31:331-341.

37 Park SG, et al: T regulatory cells maintain intestinal homeostasis by suppressing $\gamma \delta \mathrm{T}$ cells. Immunity 2010;33:791-803.

38 Spits H, Di Santo JP: The expanding family of innate lymphoid cells: regulators and effectors of immunity and tissue remodeling. Nat Immunol 2011;12:21-27.

39 Aparicio-Domingo P, Cupedo T: Ror $\gamma \mathrm{t}+\mathrm{in}$ nate lymphoid cells in intestinal homeostasis and immunity. J Innate Immun 2011;3:577584.

40 Buonocore S, et al: Innate lymphoid cells drive interleukin-23-dependent innate intestinal pathology. Nature 2010;464:13711375

41 Wolk K, et al: Biology of interleukin-22. Semin Immunopathol 2010;32:17-31.

42 Sonnenberg GF, et al: CD4(+) lymphoid tissue-inducer cells promote innate immunity in the gut. Immunity 2011;34:122-134. 